\title{
Doppler Correction of Mobile Acoustic Communication via Adjustable AD Sampling Rate
}

\author{
XIAOYANG WANG \\ College of Ocean and Earth Sciences, \\ Xiamen University \\ FUMIN ZHANG \\ School of Electrical and Computer \\ Engineering, Georgia Institute of \\ Technology
}

\author{
SIYUAN ZHENG \\ College of Ocean and Earth Sciences, \\ Xiamen University \\ AIJUN SONG \\ Department of Electrical and Computer \\ Engineering, The University of \\ Alabama
}

\author{
QIUYANG TAO \\ School of Electrical and Computer \\ Engineering, Georgia Institute of \\ Technology \\ FENG TONG \\ College of Ocean and Earth Sciences, \\ Xiamen University \\ ftong@xmu.edu.cn
}

\begin{abstract}
${ }^{1}$ This paper presents a hardware Doppler suppression method for underwater acoustic (UWA) communication with compact-sized underwater platforms such as autonomous underwater vehicles (AUVs). The proposed method adopts an adjustable AD (analogto-digital) sampling rate, leading to significant reductions in the computational complexity of the communication receiver. Both direct sequence spread spectrum (DSSS) and channel coding technology are incorporated into the proposed Doppler compensation method to design a mobile UWA communication system. Sea experiments have been conducted with moving vehicles, and the experimental results have demonstrated the effectiveness of the proposed method.
\end{abstract}

\section{KEYWORDS}

mobile UWA communication, doppler compensation,AD sampling rate

\section{ACM Reference format:}

Xiaoyang Wang, Siyuan Zheng, Qiuyang Tao, Fumin Zhang, Aijun Song,F. Tong. 2017. Doppler Compensation of Mobile Acoustic Communication via Adjustable AD Sampling Rate. In Proceedings of the 13th ACM International Conference on Underwater Networks \& Systems, December 3-5, 2018 (WUWnet'18) ,5 pages.

https://doi.org/10.1145/3291940.3291978

\footnotetext{
Permission to make digital or hard copies of all or part of this work for personal or classroom use is granted without fee provided that copies are not made or distributed for profit or commercial advantage and that copies bear this notice and the full citation on the first page. Copyrights for components of this work owned by others than ACM must be honored. Abstracting with credit is permitted. To copy otherwise, or republish, to post on servers or to redistribute to lists, requires prior specific permission and/or a fee. Request permissions from Permissions@acm.org.

WUWNet'18, December 3-5, 2018, Shenzhen, China 2018 Association for Computing Machinery. ACM ISBN 978-1-4503-6193-4/18/12.. \$15.00 https://doi.org/10.1145/3291940.3291978
}

\section{INTRODUCTION}

Mobile platforms( i.e. ,AUVs) have been developed for versatile tasks including underwater surveys, environmental monitoring, and acoustic communication experiments. UWA communication is required for many AUV applications to allow data transmission without vehicle surfacing[1]. However, multipath expansion, time-varying fading, and Doppler spreading in both the time and frequency domain are all challenges for underwater acoustic communication. Among these difficulties, Doppler spread is the most severe issue because of the relative motion between mobile platforms[2]. Significant amounts of research have been conducted on Doppler suppression. Johnson et al . [3] proposed a method for correlation between a set of local Doppler signals (with different offsets) and received signals, and the Doppler factor was calculated using the correlator with the highest correlation. Sharif et al.[4] used a linear frequency modulation (LFM) signal for Doppler estimation based on two LFM correlation peak intervals. The proposed Doppler estimator proved to be robust despite the complex channel responses. Tu et al.[5] designed a multiple-resampling (MR) scheme, where each MR branch matches to the Doppler factor of a particular propagation path.Chengbing $\mathrm{He}$ et al.[6] proposed an accurate Doppler shift estimation method,which automatically matches the underwater acoustic multipath channels. However, most existing efforts focus on improving the estimation accuracy of the Doppler factor and do not emphasize the communication efficiency in practical applications. These methods usually require a priori information and one complete signal frame. Text, the algorithms obtain the Doppler estimation by resampling or linearly calculating the entire frame signal. Unfortunately, reconstructing and resampling the frame signal is computationally intensive, which poses challenges to the onboard electronics of the smallsized underwater platforms.

In this work, we propose a hardware Doppler mitigation approach by directly tuning the $\mathrm{AD}$ sampling rate. This approach significantly reduces the computational cost and is easier to implement on small-sized underwater robots. Each signal frame begins with a synchronization signal, which also serves as the Doppler probe. Once the synchronization is completed, the AD 
sampling rate is adjusted to directly overcome the Doppler effect. Therefore, this approach is well suited to the acoustic communication of mobile underwater platforms, which favors the Doppler estimation feature but have limited computational cost.

\section{SYSTEM DESIGN AND ANALYSIS}

\subsection{DSSS Modulation}

The principle of spread spectrum (SS) technology is to modulate the information to be transmitted into a wide frequency band by a pseudo-random sequence and then recover the transmitted information sequence through the demodulation and despreading process at the receiving end. The spread spectrum technology mainly includes DSSS, FH (frequency-hopping), LFM, and some mixed spread spectra. In this study, the direct sequence spread spectrum method is adopted. The system using DSSS has strong anti-interference ability, anti-multipath capability and it can work under low SNR(signal-noise-ratio) conditions [7]. Differential coherent demodulation delays the received signal by a symbol interval (interval $=\mathrm{Ts}$ ) and then multiplies it by the original signal. After completing the demodulation, it also completes the understanding of the difference, and finally it passes the low-pass filtering to perform the sampling decision. It has the advantage that it does not need to generate a local coherent carrier, and it is difficult to estimate and track the carrier in the underwater acoustic channel. This paper uses the DS-DBPSK(direct sequency-differential binary phase shift keying) modulation method. The specific implementation block diagram is shown in Fig. 1.

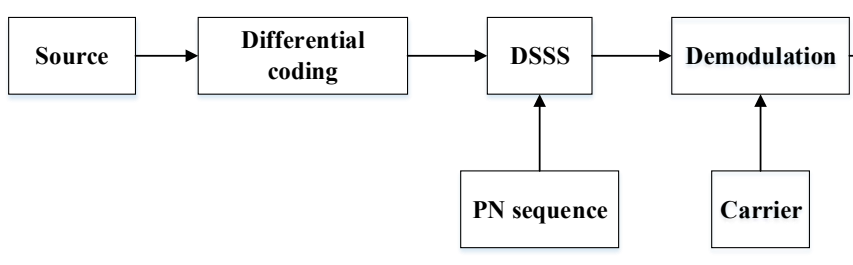

Figure 1: Block chart of DSSS modulation.

\subsection{Doppler Estimation}

In traditional time domain estimation, the Doppler shift is first calculated after receiving a complete frame signal, and then the data segment is linearly interpolated or resampled according to the estimation result. To reduce the Doppler estimation and compensation time, the signal frame format designed in this paper is shown in Fig. 2.

\begin{tabular}{|c|c|c|c|c|c|}
\hline LFM & $\begin{array}{c}\text { Protection } \\
\text { interval }\end{array}$ & $\ldots$ & LFM & $\begin{array}{c}\text { Protection } \\
\text { interval }\end{array}$ & $\begin{array}{c}\text { Data } \\
\text { segment }\end{array}$ \\
\hline
\end{tabular}

Figure 2: Structure of data packet.
The Doppler time domain expansion is equivalent to the degree of signal contraction or expansion [8], and the first and last LFM correlation peaks in the signal can be taken and the Doppler factor calculated according to Equation 1.

$$
\Delta \approx \frac{T_{r p}}{T_{t p}}-1
$$

Where $T_{r p}$ is the time domain length of received signal and $T_{\mathrm{t} p}$ is the time domain length of transmission signal.

\subsection{Doppler Compensation Based on the Adjustable AD Sampling Rate}

In the process of communication, while the receiving end seizes the signal synchronization, the Doppler shift can also be estimated. At this time, according to the estimation result, the AD sampling rate of the system can be directly adjusted, which is equivalent to performing a Doppler compensation on the data segment of the signal. Usually, the hardware sampling has only a fixed number of sampling rates through the audio chip, and the method of using the $\mathrm{AD}$ variable sampling rate is more flexible and convenient; even for small-scale offsets, the sampling performance of the system can be ensured by the high-precision timer clock. Such a Doppler compensation scheme can reduce the computational complexity of the MCU(Microcontroller-Unit) and further improve the real-time performance of the system, which is convenient for hardware implementation.

\subsection{Hardware Implementation}

Because the Doppler is directly compensated by hardware means, the proposed system is capable of greatly reducing the complexity of the calculation in the MCU, thus ensuring the communication timeliness and facilitating hardware implementation in small-size platforms such as AUVs. The implementation block diagram of the receiver is shown in Fig. 3.

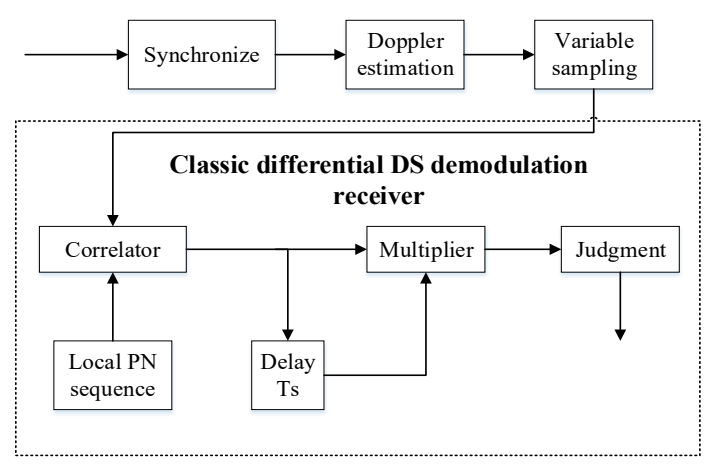

Figure 3: Receiver of SS communication system based on AD variable sampling rate.

\subsection{Analysis of Complexity}

In this paper, the duration of a frame is 6.8 seconds, the signal sampling rate is $75000 \mathrm{sps}$, and the data volume is 510000 points. The principle of traditional Doppler compensation is to resample 
the received data after the Doppler estimation. Specifically, the original signal sequence is first up-sampled by an integer factor of I, and then the output sequence is obtained by a linear interpolation algorithm according to Equation 2.

$$
y(m+1)=\left(1-I t_{m+1}\right) y_{1}(m+1)+I t_{m+1} I y_{2}(m+1)
$$

where $y(m+1)$ is the output point after resampling, $t_{m+1}$ indicates the sampling time of $y(m+1)$, and $y_{1}$ and $y_{2}$ are the adjacent points after upsampling. It can be known from the algorithm formula that the time complexity of the resampling process algorithm is $\mathrm{O}\left(\mathrm{m}^{2}\right)$. In addition, the up-sampling process increases the amount of data, and the additional space complexity of the MCU memory is $\mathrm{O}(\mathrm{m}-\mathrm{m} / \mathrm{I})$. With the proposed hardware approach, the resampling is directly performed during the $\mathrm{AD}$ sampling, thus avoiding the calculation of software resampling.

\section{EXPERIMENT INTRODUCTION}

\subsection{Experiment Setup}

The experimental sea area is the Xiamen sea area, and the configuration is shown in Fig.4,with ship A anchored and the ship B approaching ship A at a speed of 3 knots. The distance of the two ships is $1.2 \mathrm{~km}$, with a water depth of $10 \mathrm{~m}$. The sound velocity gradient is shown in Fig. 5.

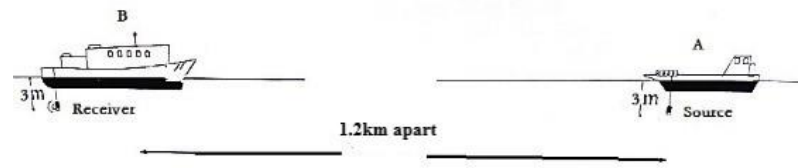

Figure 4: Configuration of sea trial.

To evaluation the performance of the method in this paper, the experimental signal transmission format is shown in Fig. 6. The previous part is the Doppler estimation segment, which contains two LFM signals with a duration of $22.6 \mathrm{~ms}$ to estimate the Doppler. The latter part involves the data segment, and the carrier frequency used for the modulation is $15.5 \mathrm{kHz}$. The transmitting end converts the string of length 48 into binary data by data type conversion, convolutional coding, differential encoding and so on, a total of 780 bits; then it performs direct sequence spread spectrum modulation by means of PN code. The experimental system designed by this research adopts the method of differential encoding first, which exhibits better anti-noise performance[9].

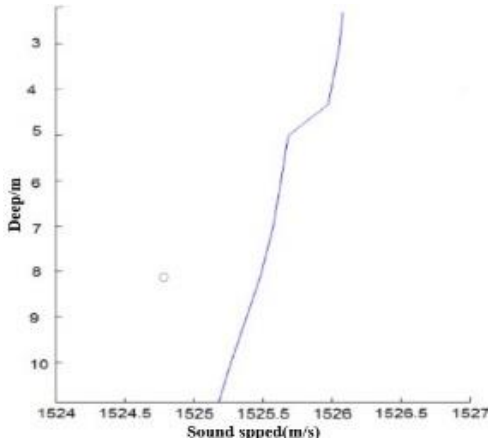

Figure 5: Sound velocity gradient.

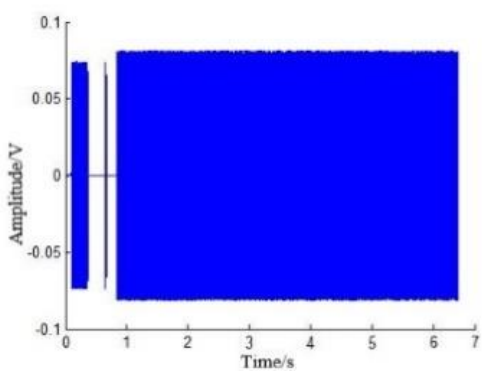

Figure 6: Structure of data packet.

\subsection{Experimental Results and Analysis}

The multipath intensity profile (MIP) of the sea trial channel is shown in Fig. 7. It can be seen that the channel has an obvious multipath. The time-varying response of the sea trial channel is shown in Fig. 8[10]. One may observe that it exhibits apparent Doppler shift caused by the relative motion of the transceiver platform, which is mainly determined by the relative motion speed, and the Doppler variation is small over this short period.

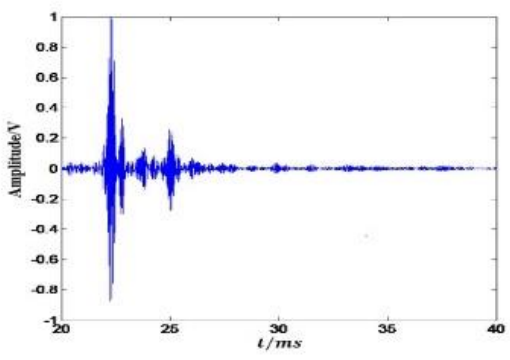

Figure 7: MIP of the channel. 


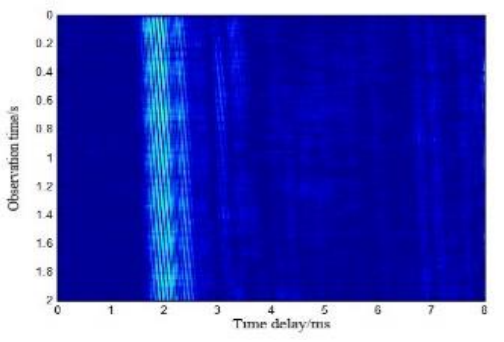

Figure 8: Time-varying channel response.

To evaluate the system performance, 10 frames of communication signals were selected for analysis by the software and hardware Doppler mitigation techniques. For the software receiver, the MATLAB software was adopted for off-lined demodulation, while the proposed STM32 MCU hardware was used for real-time hardware demodulation. The Doppler values estimated by the software and hardware are shown in Fig. 9. It can be seen that the signal frequency offset is between 5 and 20Hz.Shown in Fig. 10 is the bit error rate(BER) curve corresponding to the software and hardware receiver, wherein the solid line is a hardware demodulation error rate curve, and the broken line is a software demodulation BER curve. From the BER results, it is evident that, with a higher calculation complexity, the software demodulation performance is slightly better than hardware demodulation. The reason for the analysis may be that the precision of the hardware sampling chip, system clock, and so on. is not as good as the software.

In addition, Fig. 10 also indicates that the BER after Doppler compensation was less than 0.06 , and the communication performance was obviously better than that before compensation. Moreover, the adoption of channel code further improved the BER to the level of under 0.01. In all, incorporating the DSSS modulation and channel coding, the proposed Doppler compensation method based on the $\mathrm{AD}$ variable sampling rate achieved strong communication performance for the moving vehicle.

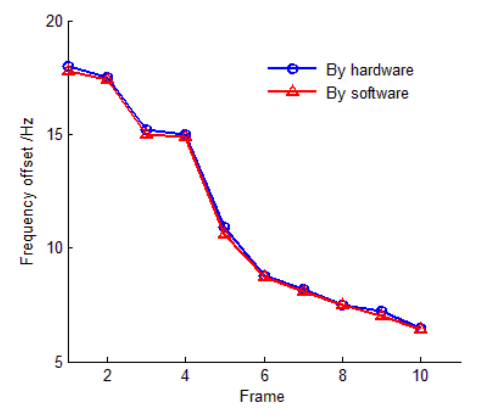

Figure 9: Doppler estimated by software and hardware method.

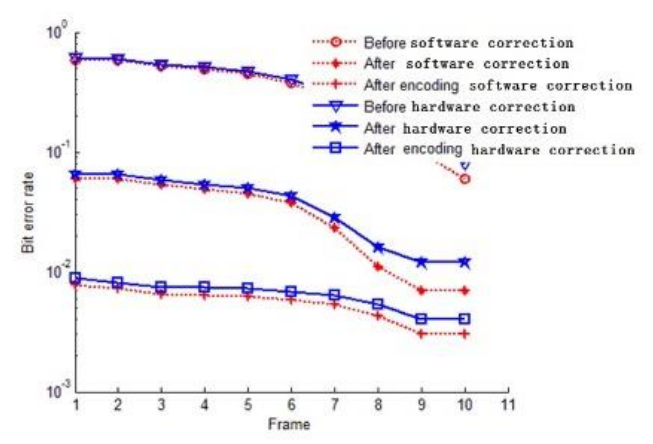

Figure 10: Error rate curves.

\section{CONCLUSIONS}

This paper presents a Doppler compensation method for smallsized AUVs based on AD variable sampling. This hardware-based method greatly reduces the complexity of implementation and enables real-time Doppler mitigation. The experimental results of the mobile communication sea experiments verified the effectiveness of the proposed approach, which can improve system performance, reduce implementation complexity, and achieve strong communication effects.

\section{A HEADINGS IN APPENDICES}

The rules about hierarchical headings discussed above for the body of the article are di.erent in the appendices. In the appendix environment, the command section is used to indicate the start of each Appendix, with alphabetic order designation (i.e., the first is $\mathrm{A}$, the second $\mathrm{B}$, etc.) and a title (if you include one). So, if you need hierarchical structure within an Appendix, start with subsection as the highest level. Here is an outline of the body of this document in Appendix-appropriate form:

\section{A.1 Introduction}

\section{A.2 SYSTEM DESIGN AND ANALYSIS}

\section{A.2.1 DSSS Modulation \\ A.2.2 Doppler Estimation Sampling Rate \\ A.2.4 Hardware Implementation \\ A.2.5 Analysis of Complexity}

A.2.3 Doppler Compensation Based on the Adjustable AD

\section{A.3 EXPERIMENT INTRODUCTION}

\section{A.3.1 Experiment Setup \\ A.3.2 Experimental Results and Analysis}

\section{A.4 Conclusions}

\section{A.5 References}




\section{ACKNOWLEDGMENTS}

The authors are grateful for funding by grants from the National Natural Science Foundation of China (No. 11274259, 11574258, 61673370), as well as the Office of Naval Research Grant (No. N00014-16-1-2667) in support of the present research.

\section{REFERENCES}

[1] Q.Y TAO, Y.H ZHOU, F.TONG, A.J SONG, F.M ZHANG, Evaluating acoustic communication performance of micro autonomous underwater vehicles in confined space[J]. Frontiers of Information Technology \& Electronic Engineering, in press

[2] N. JIA, J.C HUANG. Review of underwater acoustic communication technology[J]. Physics, 2014, 43(10):650-657.

[3] JOHNSON M, FREITAG L, STOJANVIC M. Improved Doppler tracking and correction for underwater acoustic communications[C], IEEE International Conference on Acoustics, Speech, and Signal Processing, 1997. ICASSP-97., 1997.Munich: 1997, 1: 575-578.

[4] SHARIF B S, NEASHAM J, HINTON O R, et al. A computationally efficient Doppler compensation system for underwater acoustic communications[J]. IEEE Journal of Oceanic Engineering, 2000, 25(1):52-61.

[5] TU K, DUMAN T M, STOJANOVIC M, et al. Multiple-resampling receiver design for OFDM over Doppler-distorted underwater acoustic channels[J]. IEEE Journal of Oceanic Engineering, 2013, 38(2): 333-346.

[6] C.B. HE, J.G. HUANG, Q.W. MENG et al. Accurate Estimation Method of Water Acoustic Multipath Channel Doppler Factor[J]. Electroacoustic Technology, 2010, 34(12): 57-59.

[7] L.Q. SUN. Research on time-reverse mirror spread spectrum underwater acoustic communication technology [D]. Harbin: Harbin Engineering University, 2010

[8] LI B, ZHOU S, STOJANOVIC M, et al. Pilot-tone based ZP-OFDM demodulation for an underwater acoustic channel[C], OCEANS 2006. MA: IEEE, 2006: 1-5.

[9] Y.H. ZHOU, X.L. CAO, Y.Y. WU et al. Passive time-reversed spreadspectrum underwater acoustic communication under time-varying channels[J].Applied Acoustics,2015,34(6):509-515

[10] Y.H. ZHOU,F.TONG,G.Q. ZHANG. Distributed compressed sending estimation of underwater acoustic OFDM channel[J]. Applied Acoustics,2016,117:160-166 